\title{
Applications of nonparametric adaptive methods for simple reaction time experiments
}

\author{
Yung-Fong Hsu ANd Yen-Ho Chen \\ National Taiwan University, Taipei, Taiwan
}

\begin{abstract}
Adaptive methods are commonly used in psychophysical research for detection and discrimination (see Leek, 2001; Treutwein, 1995, for reviews). In recent years, researchers have investigated via simulations some asymptotic and small-sample properties of two nonparametric adaptive methods - namely, the fixed-step-size up-down (García-Pérez, 1998, 2001) and the (accelerated) stochastic approximation (Faes et al., 2007). In the present article, we extend both methods to the simple reaction time (RT) situation for the measure of signal intensities that elicit certain (fixed) RT percentiles. We focus on extending the following four methods: the stochastic approximation of Robbins and Monro (1951), its accelerated version of Kesten (1958), the transformed up-down of Wetherill (1963), and the "biased coin design" of Durham and Flournoy $(1994,1995)$. In all simulations, we assume that the RT is Weibull distributed and that there is a linear relationship between the mean RT and its standard deviation. The convergences of the asymptotic and small-sample properties for different starting values, step sizes, and response criteria are systematically investigated.
\end{abstract}

It has been common in reaction time (RT) experiments to use the ANOVA to analyze means across covariate conditions. For example, the Stroop task (Stroop, 1935) employs the nominal covariates of a color word and the text color in which it is written, and mean RTs of covariate categories are the objects analyzed (see MacLeod, 1991, for a review). However, when dealing with continuous covariates, such as age, IQ, stimulus intensity, symbolic distance, word frequency, and so on, the ANOVA approach - with its categorized means - tends to ignore the potentially rich relations between covariates and RTs. To remedy this situation, researchers have started to develop alternative, process-based methodologies for RT analysis. For example, Rouder and colleagues (Rouder, Lu, Speckman, Sun, \& Jiang, 2005; Rouder, Sun, Speckman, Lu, \& Zhou, 2003) have provided a general Bayesian framework to model RT (distributions) as a function of continuous covariates.

In the present article, we challenge the nominal-covariate approach for continuous covariates in a within-subjects design from a different perspective. We provide a principled method for integrating continuous covariates into RT modeling. The idea originated from the adaptive methods commonly used in psychophysical research for detection and discrimination. We introduce the notion of "sensitivity" and describe the algorithms to estimate it. In principle, this method can be applied to any continuous covariate. For instance, to study the relationship between word frequency and naming times in the so-called "go/no-go" experimental paradigm, in which subjects are instructed to press a button if the presented string is an English word but to refrain from pressing if the presented string is a nonword (see, e.g.,
Gomez, Ratcliff, \& Perea, 2007), one may use the method to estimate the word frequency needed to elicit a certain RT at a given percentile. ${ }^{1}$

In the following section, we will briefly describe a "sensitivity function" in the context of a simple RT experiment and a commonly studied continuous covariate: the signal intensity/contrast. We will also motivate our development from a small-sample perspective, arguing that our method is an appropriate RT design for estimating the sensitivity function efficiently.

\section{Simple RT}

In a standard simple RT experiment, a trial starts with a warning signal, followed by a foreperiod, at the end of which the signal is presented. The participant is required to react to the presence of the signal as quickly as possible (Hsu, 2005b; Luce, 1986).

Historically, Piéron's law has been one of the most widely cited functional forms specifying the relation between the RT and the signal intensity $i$ (Luce, 1986):

$$
\text { Mean or Median } \operatorname{RT}(i)=R+i^{-\beta} K \text {, }
$$

in which $R, \beta$, and $K$ are positive parameters. ${ }^{2} \mathrm{Hsu}$ (2005a) extended the above equation to RT percentiles $T_{\pi}$ (given a fixed background). For all $\pi(0<\pi<1)$,

$$
T_{\pi}(i)=R(\pi)+i^{-\beta(\pi)} K(\pi),
$$

from which one sees that the parameters $R, \beta$, and $K$ are now functions of the probability $\pi .^{3}$

Influenced by the seminal work of Falmagne (1985) on the near miss to Weber's law in psychophysics for detec- 
tion and discrimination, Hsu (2005a) introduced a sensitivity function $\xi(\tau, \pi)$, which is the signal intensity that elicits a $\tau$-msec "response" for the $(\pi \times 100)$ th RT percentile (given a fixed background), suggesting a switch of focal interest. Instead of focusing on the RT percentiles expressed in the generalized Piéron's law, one can study the signal intensity via the following equation:

$$
\xi(\tau, \pi)=\exp \left[\frac{\log \frac{\tau-R(\pi)}{K(\pi)}}{-\beta(\pi)}\right] .
$$

Thus, there is an issue of how to obtain a reliable estimate of the sensitivity function $\xi$. In the present article, we sometimes call $\xi$ the "target" or "(target) quantile" when there is no ambiguity.

\section{Small Samples}

Besides the aim of integrating continuous covariates into RT modeling, the present research was motivated by the fact that analyzing RTs at the distribution level requires a large number of observations (per subject) to be reliable and effective. However, collecting RT data using the standard experimental procedure is time consuming. Furthermore, very often researchers have some distributional assumptions for RTs, for which there is a mandate to obtain reliable estimates of the parameters. Such a work may be challenging if the sample size is small. In the past, researchers have explored various statistical modeling approaches to tackle this problem. An excellent example of work along this line is the hierarchical Bayesian modeling framework that was developed by Rouder and colleagues (Rouder et al., 2005; Rouder et al., 2003; Rouder, Tuerlinckx, Speckman, Lu, \& Gomez, 2008). The adaptive method we advocate present for RT experiments, if indeed feasible, will be especially useful for testing participants who can only contribute a small amount of data.

For example, in recent years, there have been advances in using adaptive methods for perceptual learning to improve contrast detection and discrimination of adults and children with certain visual impairment, such as amblyopia and blindsight (see, e.g., Huang, Zhou, \& Lu, 2008; Polat, Ma-Naim, Belkin, \& Sagi, 2004; Sahraie et al., 2006; Zhou et al., 2006). We believe that for those people, it is also important to improve the speed of discrimination for above-threshold contrasts/objects. They may benefit from such evaluation and training, because being able to react to surrounding contrasts/objects quickly is also crucial for their everyday lives. Such a program could only be executed with an appropriate experimental design involving RT, and our method has the potential to help achieve this goal.

The present article is organized as follows. First, we will give a formal introduction to the application of adaptive methods for RT experiments. The concepts of the two adaptive methods that we are to explore - the stochastic approximation and the fixed-step-size up-down methods - will be briefly reviewed. Then, we will specify the setting for all the simulations. In particular, we will assume a family of three-parameter Weibulls for the RT distributions. Some concerns are dedicated to spelling out the conditions for simulating the behavior of RTs that match closely those from empirical findings. We will devote a section to the main results. We will perform a series of simulations aimed at exploring the asymptotic and small-sample properties of both methods by varying the starting values, step sizes, and response percentiles/ criteria systematically.

\section{Adaptive Methods for RT Experiments}

We are concerned with obtaining an efficient estimate of the signal intensity that elicits a certain RT percentile. The adaptive methods commonly used in the detection and discrimination studies in psychophysics can be extended to the RT situation to suit this goal.

In adaptive methods, the presentation of the stimulus on the current trial depends on the response the subject made on the preceding trial(s). Probably because of their efficiency, currently, adaptive methods are preferable to classical methods, such as the method of constant stimuli and the method of adjustment, for estimating the sensory threshold or the just-noticeable difference. (see Falmagne, 1985; Leek, 2001; Treutwein, 1995, for reviews).

Both parametric and nonparametric adaptive methods, with the difference being whether there is a distributional assumption of the underlying psychometric function, have been developed extensively in the psychophysical literature (Leek, 2001; Treutwein, 1995). In the present article, we focus on the nonparametric adaptive methods, because of their simplicity. The reader is also referred to Treutwein (1995) or Falmagne (1985) for a mathematically oriented introduction to this subject in the framework of psychophysics. In recent years, researchers have investigated via simulations some asymptotic and small-sample properties of two popular nonparametric adaptive methods: the fixed-step-size up-down (García-Pérez, 1998, 2001), which includes the transformed up-down and other variants, and the (accelerated) stochastic approximation (Faes et al., 2007).

In the following section, we will extend the above two nonparametric adaptive methods to the simple RT situation. Specifically, instead of measuring the participant's RTs for a given signal, as was usually done in the traditional simple RT study, we set a fixed latency $\tau$ and estimated the signal intensity that elicits a certain RT percentile. We focused on the description of the stochastic approximation of Robbins and Monro (1951), its accelerated version of Kesten (1958), the transformed up-down of Wetherill (1963) and Levitt (1971), and the up-down involving random draw (the so-called "biased coin design") of Durham and Flournoy $(1994,1995)$. Although the core concepts of those methods in the RT framework follow closely those for estimating quantiles of psychometric functions in detection and discrimination, for which the references are abundant, we will give a brief review of those concepts for completeness. 
The stochastic approximation method for RT. We will consider two collections of positive random variables:

$$
\begin{aligned}
& \mathbf{X}_{n} \text { denotes the intensity of the stimulus on trial } n \text {; } \\
& \mathbf{T}_{n} \text { denotes the latency of the response on trial } n \text {. }
\end{aligned}
$$

Consider a function $F:(t ; x) \mapsto F(t ; x)$ that is a distribution function in $t$ for each fixed intensity $x$. We assume that $F(t ; x)$ is continuous and strictly increasing in both variables. Let $\xi$ be a positive real number representing a fixed intensity, and $\pi \in] 0,1[$. Moreover, let $\tau$ be positive real number representing a fixed latency. We define

$$
\mathbf{Y}_{n}= \begin{cases}0 & \text { if } \mathbf{T}_{n}>\tau, \\ 1 & \text { if } \mathbf{T}_{n} \leq \tau .\end{cases}
$$

We will now consider the (stationary) stochastic process $\left(\mathbf{X}_{n}, \mathbf{T}_{n}\right), n=1,2, \ldots$, and suppose that the following conditions are satisfied:

[R1] The function $F(\tau ; \xi)=\pi$. Moreover, $F(\tau ; x)$ is differentiable in the second variable for $x=\xi$; that is, $F^{\prime}(\tau ; \xi)>0$.

[R2] We set $\mathbf{X}_{1}=x_{1}$, an arbitrary intensity. Moreover, for $n=1,2, \ldots$,

$$
\mathbf{X}_{n+1}=\mathbf{X}_{n}-\frac{c}{n}\left(\mathbf{Y}_{n}-\pi\right),
$$

where $c>0$ is a constant.

[R3]

$$
\begin{aligned}
P\left(\mathbf{T}_{n} \leq \tau \mid \mathbf{X}_{n}, \mathbf{T}_{n-1}, \mathbf{X}_{n-1}, \ldots, \mathbf{T}_{1}, \mathbf{X}_{1}\right) & =P\left(\mathbf{T}_{n} \leq \tau \mid \mathbf{X}_{n}\right) \\
& =P\left(\mathbf{Y}_{n}=1 \mid \mathbf{X}_{n}\right) \\
& =F\left(\tau ; \mathbf{X}_{n}\right) .
\end{aligned}
$$

The following theorem is originally due to Robbins and Monro (1951):

Theorem. Under Conditions [R1], [R2], and [R3], we have

$$
\lim _{n \rightarrow \infty} E\left(\mathbf{X}_{n}-\xi\right)^{2}=0 .
$$

That is, the sequence of random variables $\left(\mathbf{X}_{n}\right)$ converges to $\xi$ in mean square, and hence in probability.

Recall that a sequence of random variables $\left(\mathbf{X}_{n}\right)$ converges to a random variable $\mathbf{X}$, if for every $\epsilon>0$

$$
\lim _{n \rightarrow \infty} P\left(\left|\mathbf{X}_{n}-\mathbf{X}\right|>\epsilon\right)=0 .
$$

In view of the above theorem, one sees that the random variable $\mathbf{X}$ has all its mass concentrated at $\xi$. This result implies that the intensity value $\xi$, corresponding to an RT value $\tau$ and a probability $\pi$, can be estimated by this method. We can thus estimate a function $(\tau, \pi) \mapsto \xi(\tau, \pi)$. We refer to this stochastic approximation method as "SA."

Kesten (1958) proposed an accelerated version of the stochastic approximation method by permitting the step size to be changed only when a shift in the response category occurs. He proved the convergence in probability of such a sequence. The modification to the RT situation is straightforward. Specifically, for the first two trials, the SA (cf. Equation 2) is used; afterward, we replace (2) with

$$
\mathbf{X}_{n+1}=\mathbf{X}_{n}-\frac{c}{n_{\text {shift }}+2}\left(\mathbf{Y}_{n}-\pi\right), n>2,
$$

where $n_{\text {shift }}$ is the number of shifts (or reversal points) in the response category. We refer to this accelerated version as "ASA."

The up-down method for RT. Another family of nonparametric adaptive methods is the fixed-step-size up-down (or "staircase") method, which has been used extensively in psychophysical research for detection and discrimination (García-Pérez, 1998; Levitt, 1971; Treutwein, 1995). We start with the simple up-down method of Dixon and Mood (1948), which can be described by replacing (2) with

$$
\mathbf{X}_{n+1}=\mathbf{X}_{n}-\delta\left(2 \mathbf{Y}_{n}-1\right),
$$

where $\delta$ is the fixed step size. ${ }^{4}$ Together with the assumptions in [R1] and [R3], it can be shown that (5) defines a recurrent Markov chain (actually, a random walk), and the distribution of $\mathbf{X}_{n}$ converges as $n \rightarrow \infty$. With some mild side conditions, it can be proved that the estimate from such a sequence yields the 50th RT percentile for a given latency $\tau$.

The simple up-down method can be extended to other nonmedian RT quantiles, and several algorithms have been developed in the literature. There are two noteworthy examples. One is the well-known transformed up-down (TRUD, also known as "UDTR") by Wetherill (1963) (see also Levitt, 1971; Wetherill, Chen, \& Vasudeva, 1966), and the other is the up-down involving "random draw" by Derman (1957) and later by Durham and Flournoy (1994). The reader is referred to either Giovagnoli and Pintacuda (1998) or Oron (2007) for more detailed discussions under a larger framework. In the present study, we consider these two extensions. ${ }^{5}$

Extending the simple up-down method, one can derive TRUD for RT using a similar logic as that in psychophysics to obtain the signals eliciting certain nonmedian RT percentiles. In this method, for $0.5<\pi<1$, the change of stimulus on the current trial depends on the outcomes of consecutive fast $(\leq \tau)$ RT responses on two or more preceding trials, yielding, for example, signals corresponding to the 70.7 th $(1-$ up/2-down) or 79.4 th $(1-$-up/ 3 -down) RT percentiles. Formally, let $k \geq 2$ be the number of consecutive trials required by the task for fast $(\leq \tau)$ responses to the given signal. In view of (1) and generalizing (5), we set $\mathbf{X}_{1}=x_{1}$; for $n=1, \ldots, k-1, \mathbf{X}_{n+1}=\mathbf{X}_{n}$ if $\mathbf{Y}_{n}=1$, and $\mathbf{X}_{n+1}=\mathbf{X}_{n}+\delta$ otherwise. Furthermore, for $n=k, \ldots$,

$$
\mathbf{X}_{n+1}= \begin{cases}\mathbf{X}_{n}+\delta & \text { if } \mathbf{Y}_{n}=0, \\ \mathbf{X}_{n}-\delta & \text { if } \mathbf{Y}_{n}=\ldots=\mathbf{Y}_{n-k+1}=1 \\ & \text { and } \\ & \mathbf{X}_{n}=\ldots=\mathbf{X}_{n-k+1}, \\ \mathbf{X}_{n} & \text { in all other cases. }\end{cases}
$$




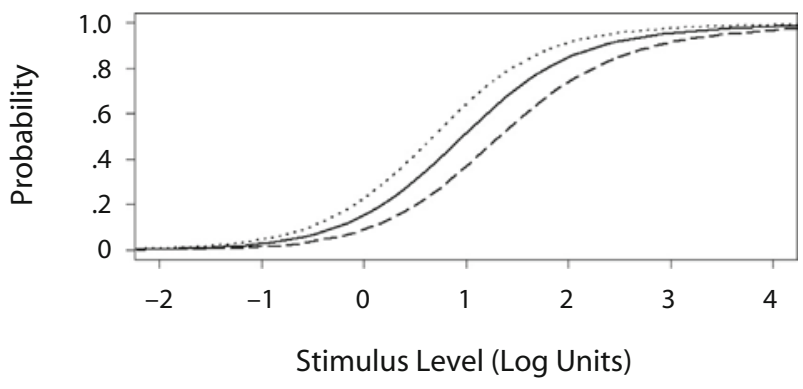

Figure 1. A generic graph showing the reaction time percentiles resulting from different signal intensities (in log scales), for fixed latencies of 275 (dotted curve), 250 (solid curve), and 225 (dashed curve) msec, using the settings for the family of Weibulls specified in the text.

A similar algorithm can be used to estimate the signals that elicit lower RT percentiles, such as the 29.3rd (2-up/ 1-down) or 20.6th (3-up/1-down) RT percentiles.

Durham and Flournoy $(1994,1995)$ extended the simple up-down method to any other nonmedian quantiles using a so-called "biased-coin design" (BCD). ${ }^{6}$ They proposed the following algorithm for the estimate of quantiles. In view of (1), for any choice of $0.5 \leq \pi<1$ :

$$
\mathbf{X}_{n+1}=\mathbf{X}_{n}-\delta\left[\mathbf{Y}_{n}\left(\mathbf{S}_{\pi}+1\right)-1\right]
$$

where $\mathbf{S}_{\pi}$ is a Bernoulli random variable (independent of $\left.\mathbf{Y}_{n}\right)$ taking value 1 with the probability $(1-\pi) / \pi$, and value 0 with the probability $1-(1-\pi) / \pi$. In other words, it is similar to the simple up-down, except that if the subject responds with a fast $(\leq \tau)$ RT, then the signal on the next trial is determined using a random draw: with the probability $(1-\pi) / \pi$, the signal goes down one level (i.e., $\delta$ ), and with the probability $1-(1-\pi) / \pi$, the signal stays at the same level. The final value can be estimated by choosing the mode or by averaging the modes (if there are more than one) of those quantiles in the simulation run. The modification is apparent for $0<\pi \leq 0.5$ : After a fast $(\leq \tau)$ RT, the signal on the next trial goes down one level; however, after a slow $(>\tau)$ RT, the signal on the next trial goes up one level with the probability $\pi /(1-\pi)$, and it stays at the same level with probability $1-\pi /(1-\pi)$.

Regarding the estimation of the target quantile using the fixed-step-size up-down method, various researchers have shown that the most frequent value (i.e., the mode) converges to the target or differs from the target by at most one "level" (Derman, 1957; Durham \& Flournoy, 1994; Giovagnoli \& Pintacuda, 1998). However, as Oron (2007) pointed out, numerical studies have shown that the empirical mode does not always approximate the target, and that averaging values in the sequence provides a more precise estimate. In fact, in the literature of TRUD, the final estimated value is customarily approximated by averaging the so-called "reversal points," excluding the first few. Our simulations also have shown that there is a relatively large and asymmetrical variability of the estimated mode as compared with the estimate using the averaged reversal points. Thus, in the present study, for the two fixed-stepsize up-down methods (TRUD and BCD), as in GarcíaPérez (1998, 2001), we average the reversal points (excluding the first two) for the estimate of quantiles.

\section{Simulation Design}

Both the stochastic approximation method and the fixedstep-size up-down method described previously point to a new venue of experimental design for RT. Instead of measuring the participant's RTs for a given signal, as was done in the traditional RT study, we estimate the signal intensity that elicits a certain RT percentile, for a fixed latency. Such a method is adaptive, in that the signal intensity on the current trial is adjusted on the basis of the participant's RTs on the preceding trial(s), using a response criterion $\pi$ embedded in a given algorithm specified (implicitly or explicitly) in Equations 2, 4, 6, or 7. To our knowledge, no one has systematically studied these nonparametric adaptive methods for RT experiments. In the present study, we perform a series of simulations aiming at exploring the asymptotic and small-sample properties of the aforementioned methods.

Note that typical RT distributions are unimodal and are almost always skewed to the right. Also, the mean and standard deviation of RTs are approximately linearly re-

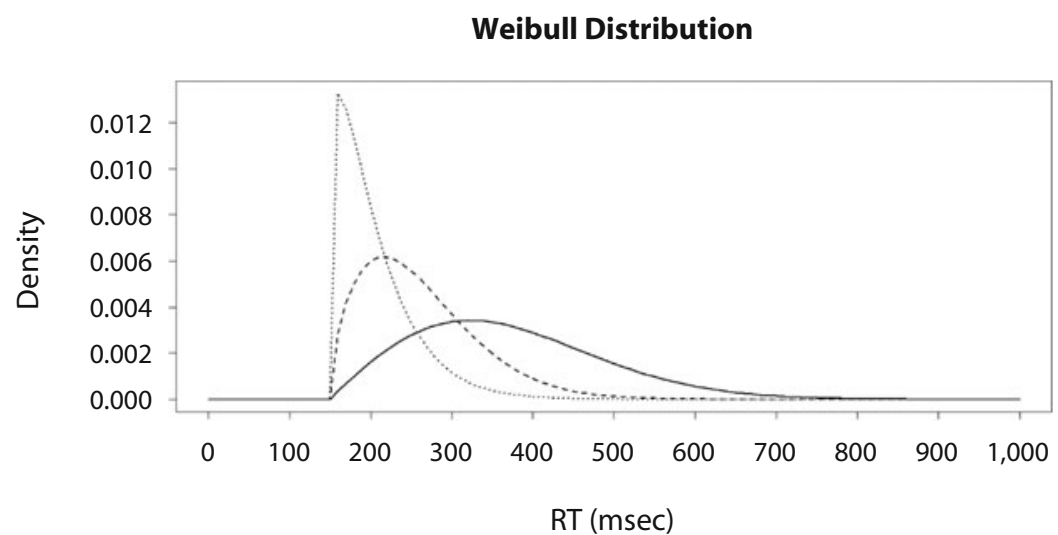

Figure 2. Weibull distributions derived from the settings $R=150, \beta=0.3, K=220$, and a ratio of 0.22 for the standard deviation to the mean reaction time (RT), for signals of 0 (solid curve), 1 (dashed curve), and 2 (dotted curve) log units. 
lated (Luce, 1986; Wagenmakers \& Brown, 2007). Thus, when using the adaptive method for a fixed latency, the corresponding change of RT percentiles is more sensitive to the change of signals if one sets the response criterion to lower percentiles than to higher percentiles. Using a $\log$ unit for the signal intensity can bypass this problem. In fact, in the literature, logarithmic scales (such as dB) are commonly adapted as the appropriate unit for equal sensory intervals. In the RT situation, we show in Figure 1 generic curves ${ }^{7}$ showing the RT percentiles resulting from different signal intensities (in log scales) using the settings for the family of Weibulls (along with other assumptions) specified in the next subsection, for fixed latencies of 275 (dotted curve), 250 (solid curve), and 225 (dashed curve) msec. An inspection suggests that the shape of each of the curves matches closely those typically seen in the study of adaptive methods in psychophysics. Accordingly, in all simulations, we take the logarithmic scale of signal intensity as the unit for the starting values and step sizes.

In the following series of simulations, we will study the asymptotic and small-sample properties of each of the four methods described previously. In particular, we will investigate systematically the impact of starting values, step sizes, and response percentiles/criteria on the behavior of sequences of trials. We will now describe the setup of the simulations.

Weibull distribution for RTs. The Weibull distribution is very flexible and is one of the commonly used forms for the underlying psychometric function. For example, the Weibull-distributed psychometric function was conveniently assumed in the simulation studies of García-Pérez $(1998,2001)$ for testing the fixed-step-size up-down method and of Faes et al. (2007) for testing the (accelerated) stochastic approximation method. In the present study, the underlying RT distribution is assumed to be a Weibull. ${ }^{8}$ Such an assumption is also theoretically motivated (see, for example, Hsu, 2005b; Rouder et al., 2005; Rouder et al., 2008, for details). Specifically, we assume a three-parameter Weibull distribution with density

$$
f(t)=a b^{-a}(t-R)^{a-1} \exp \left[-\left(\frac{t-R}{b}\right)^{a}\right] \quad(t>R),
$$

where $b$ is the scale parameter, $a$ the shape parameter, and $R$ the shift parameter. The mean of the Weibull is given by $R+b \Gamma(1+1 / a)$, and the standard deviation is given by

$$
b \sqrt{\Gamma\left(1+\frac{2}{a}\right)-\Gamma^{2}\left(1+\frac{1}{a}\right)},
$$

where $\Gamma$ is the gamma function:

$$
\Gamma(x)=\int_{0}^{\infty} t^{x-1} e^{-t} d t .
$$

To simulate the behavior of RTs faithfully, we make two more assumptions. First, it is known that RTs vary systematically with the signal intensity (Luce, 1986). As was mentioned in the introduction, it is reasonable to assume Piéron's law:

$$
\text { Mean } \mathrm{RT}(i)=R+i^{-\beta} K \text {. }
$$

Second, empirical studies have shown that the mean and standard deviation of most RT distributions are approximately linearly related (Luce, 1986; Wagenmakers \& Brown, 2007). With these two extra assumptions, we can re-express the scale and shape parameters in the Weibull as a function of the mean RT, which in turn can be written as a function of the signal intensity via (8), generating a family of Weibull-distributed RT random variables. Such an algorithm was implemented throughout the simulations.

In the present study, we set $R=150, \beta=0.3, K=220$, and 0.22 for the ratio of the standard deviation to the mean RT. Preliminary studies have shown that these parameter values yield distributions that closely match those from the empirical findings in the simple RT situation. An illustration of the Weibull distributions using this setting for signal intensities of 0,1 , and $2 \log$ units is displayed in Figure 2.

We will proceed with the general simulation procedure as follows. Using the combinations of settings described previously, in any given simulation, the signal intensity on trial $n$ is entered into the algorithm to obtain a realization of $F\left(\tau ; \mathbf{X}_{n}\right)$ (see Equation 3), which is then compared with a random number drawn from a uniformly distributed random variable, $\mathbf{U}_{n}$, in the range ]0, 1[. Specifically,

$$
\mathbf{Y}_{n}= \begin{cases}0 & \text { if } \mathbf{U}_{n}>F\left(\tau ; \mathbf{X}_{n}\right), \\ 1 & \text { if } \mathbf{U}_{n} \leq F\left(\tau ; \mathbf{X}_{n}\right) .\end{cases}
$$

The simulated value is then plugged into Equation 2, 4, 6, or 7 to determine the signal intensity used on trial $n+1$.

As was mentioned previously, in the study of adaptive methods in psychophysics (Treutwein, 1995), logarithmic scales (such as $\mathrm{dB}$ ) are commonly adopted as the appropriate unit for equal sensory intervals. Accordingly, in all simulations, we use the logarithm of the signal intensity as the unit for the starting values as well as for the step sizes. Such an implementation also allows our study to match closely those detection and discrimination studies in psychophysics.

Starting values, step sizes, and response criteria. In the simulation, we use the 20.6th, 29.3rd, 50th, 70.7th, and 79.4th RT percentiles for the response criterion $\pi$. These percentiles are chosen for practical purposes: They match the criteria corresponding to the common thresholds estimated using TRUD, and thus allow us to compare the efficiency of different adaptive methods.

We use 225, 250, and $275 \mathrm{msec}$ for the fixed latency $\tau$. These values are within a reasonable range of RT distributions covered in our initial settings (cf. Figure 2). Furthermore, we use $-1,0,1,2$, and 3 , all in log units, for the starting values $\mathbf{X}_{1}=x_{1}$. These values cover a wide range of RT percentiles and thus reflect faithfully what would happen in practice. For example, using the aforementioned setting for a fixed $\tau=250 \mathrm{msec}$, the five starting values correspond to, respectively, the 2.8th, 15.4th, 51.4th, 84.6th, and 95.4th RT percentiles.

In all of the simulations, the step sizes - namely, $c$ in Equations 2 and 4 and $\delta$ in Equations 6 and 7 -are varied systematically from 0.1 to 2 (in log units) in increments of $0.1 \log$ unit. In the literature, various researchers have 
SA

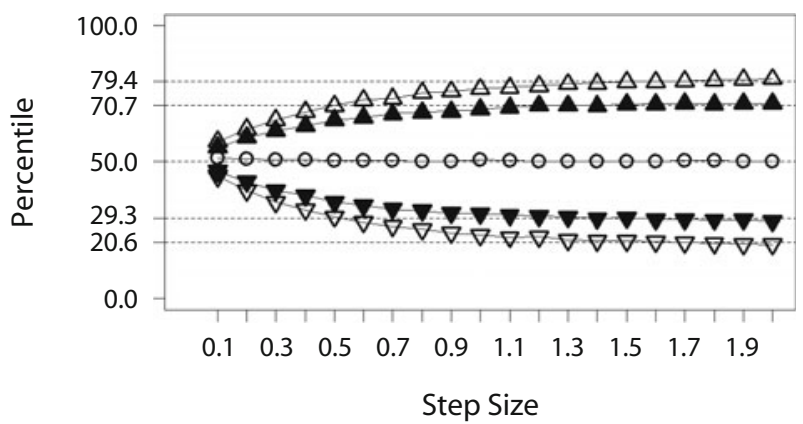

TRUD

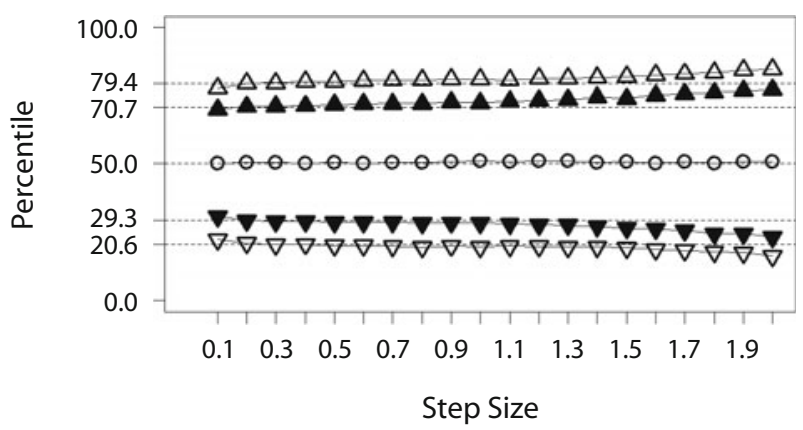

ASA

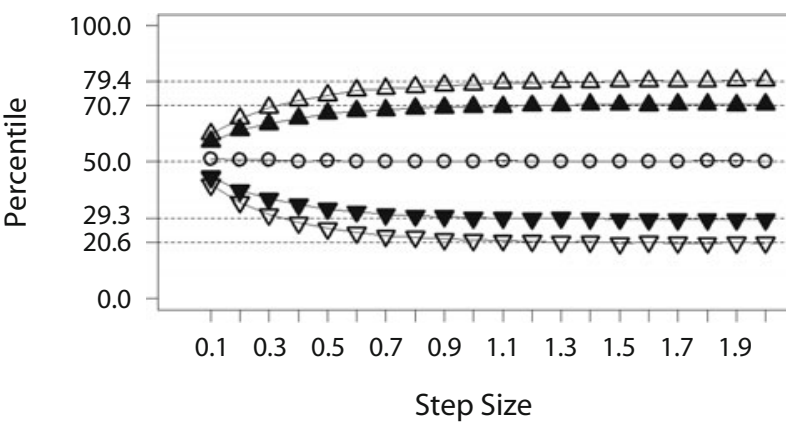

BCD

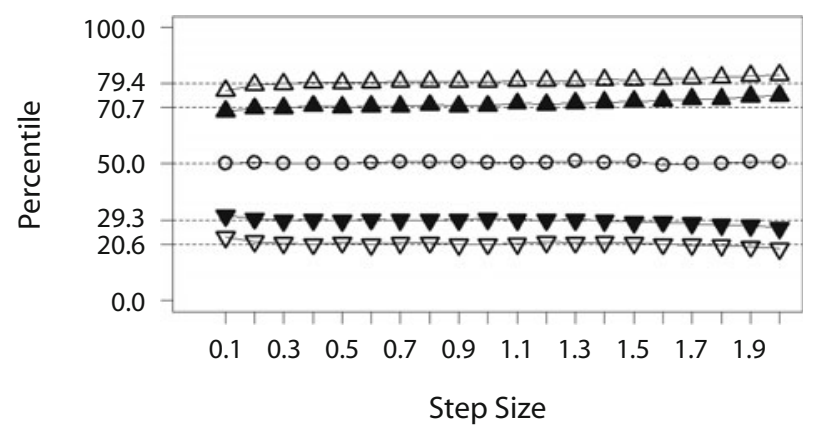

Figure 3. Convergences of stochastic approximation (SA), accelerated stochastic approximation (ASA), transformed up-down (TRUD), and the biased-coin design (BCD), based on $N=150$ trials, with a starting value at 1 log unit for the 20.6th (inverted triangles), 29.3rd (inverted filled triangles), 50th (circles), 70.7th (filled triangles), and 79.4th (triangles) reaction time percentiles/criteria, given a fixed $\tau=250 \mathrm{msec}$. For SA and ASA, the values on the $x$-axis represent the step size $c$ in Equations 2 and 4; for TRUD and BCD, they represent the step size $\delta$ in Equations 6 and 7.

SA

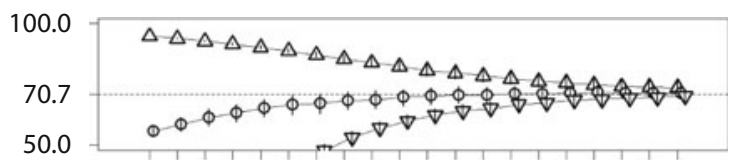

$\begin{array}{lllllllllll}0.1 & 0.3 & 0.5 & 0.7 & 0.9 & 1.1 & 1.3 & 1.5 & 1.7 & 1.9\end{array}$

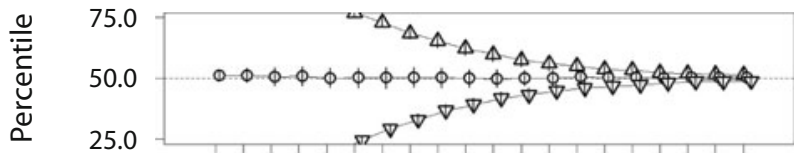

$\begin{array}{llllllllll}0.1 & 0.3 & 0.5 & 0.7 & 0.9 & 1.1 & 1.3 & 1.5 & 1.7 & 1.9\end{array}$

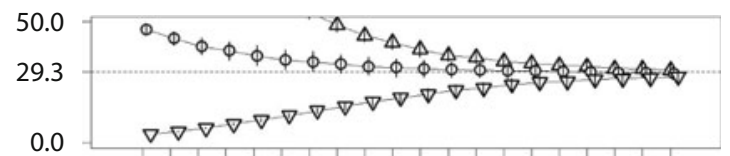

$\begin{array}{llllllllll}0.1 & 0.3 & 0.5 & 0.7 & 0.9 & 1.1 & 1.3 & 1.5 & 1.7 & 1.9\end{array}$

Step Size
ASA

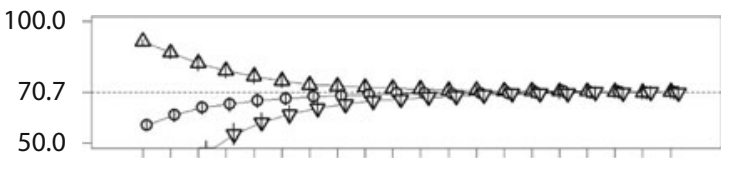

$\begin{array}{lllllllllll}0.1 & 0.3 & 0.5 & 0.7 & 0.9 & 1.1 & 1.3 & 1.5 & 1.7 & 1.9\end{array}$

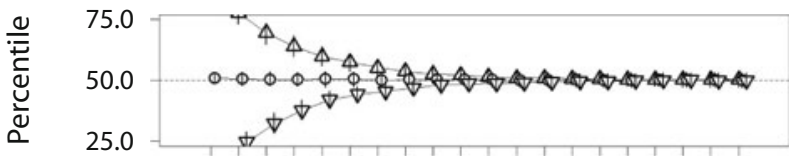

$\begin{array}{llllllllll}0.1 & 0.3 & 0.5 & 0.7 & 0.9 & 1.1 & 1.3 & 1.5 & 1.7 & 1.9\end{array}$

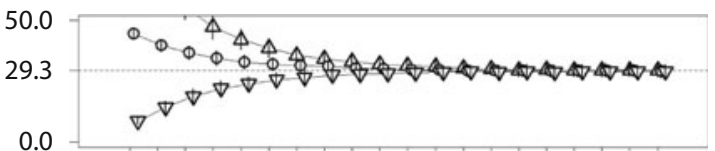

$\begin{array}{llllllllll}0.1 & 0.3 & 0.5 & 0.7 & 0.9 & 1.1 & 1.3 & 1.5 & 1.7 & 1.9\end{array}$

Step Size

Figure 4. Convergences of stochastic approximation (SA) and accelerated stochastic approximation (ASA), based on $N=150$ trials, with starting values of 3 (triangles), 1 (circles), and -1 (inverted triangles), all in log units, for the 70.7th (top panels), 50th (middle panels), and 29.3rd (bottom panels) reaction time percentiles/criteria, given a fixed $\tau=250 \mathrm{msec}$. The values on the $x$-axis represent the step size $c$ in Equations 2 and 4. Each estimate is represented by the median of 1,000 runs, with the vertical bar representing the interquartile range. Note that all the points are displaced slightly vertically so that they do not overlap. 
TRUD
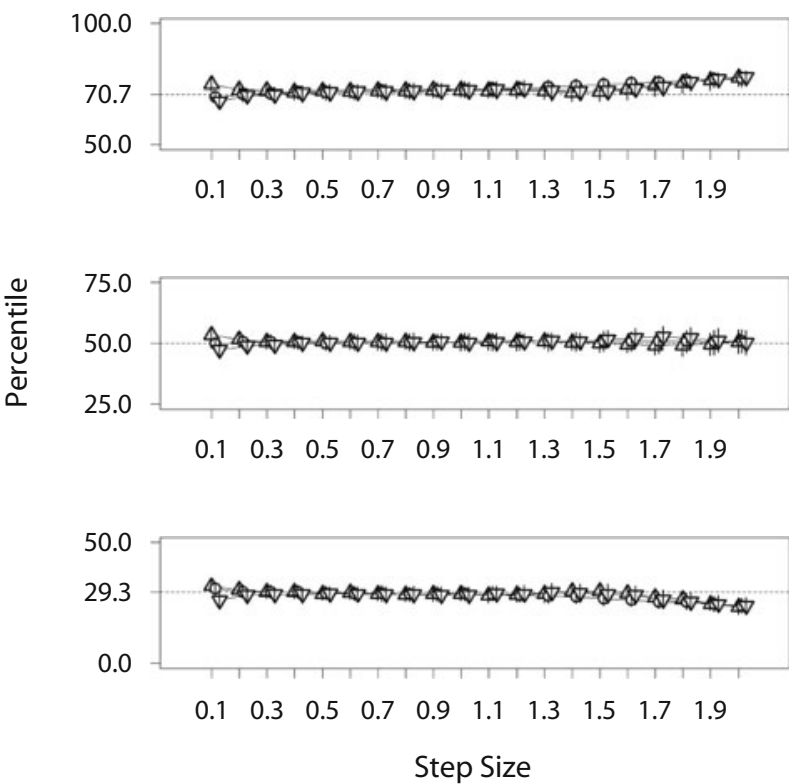

BCD
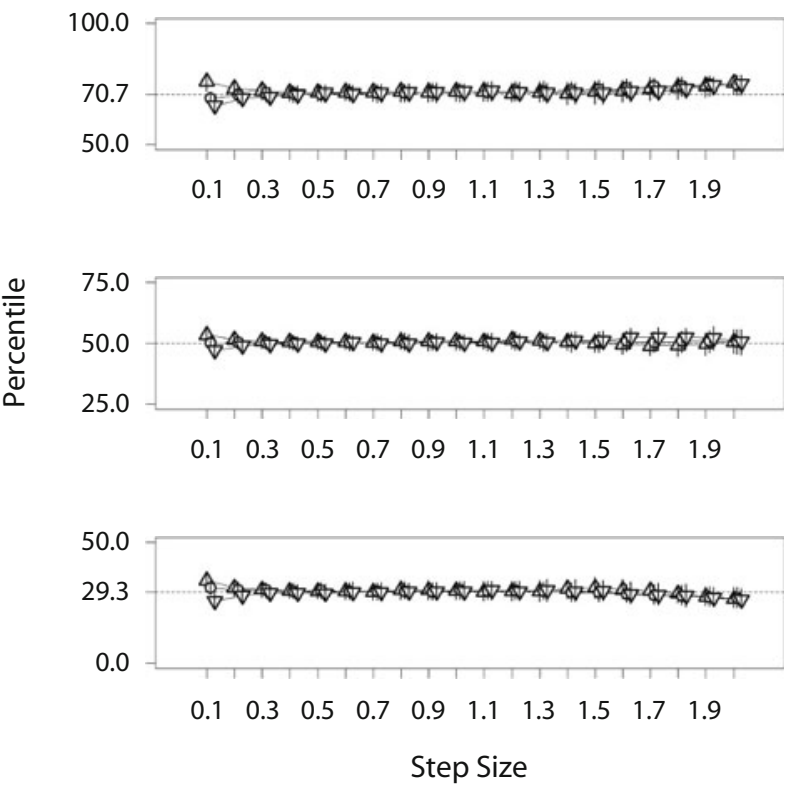

Figure 5. Convergences of transformed up-down (TRUD) and biased-coin design (BCD), based on $N=150$ trials, with starting values of 3 (triangles), 1 (circles), and -1 (inverted triangles), all in log units, for the 70.7th (top panels), 50th (middle panels), and 29.3rd (bottom panels) reaction time percentiles/criteria, given a fixed $\tau=250 \mathrm{msec}$. The values on the $x$-axis represent the fixed step size $\delta$ in Equations 6 and 7. Each estimate is represented by the median of 1,000 runs, with the vertical bar representing the interquartile range. Note that all the points are displaced slightly vertically so that they do not overlap.

SA
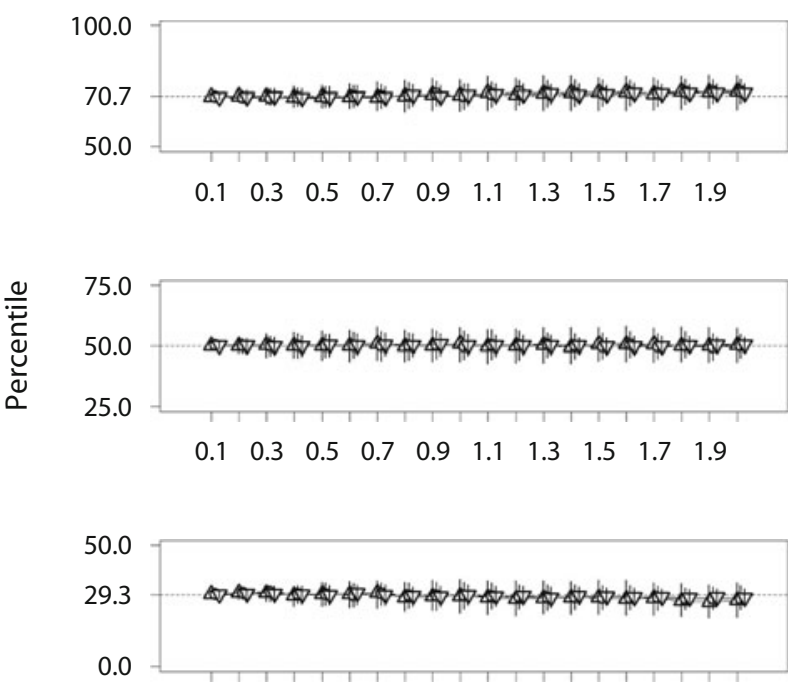

$\begin{array}{llllllllll}0.1 & 0.3 & 0.5 & 0.7 & 0.9 & 1.1 & 1.3 & 1.5 & 1.7 & 1.9\end{array}$

Step Size
ASA

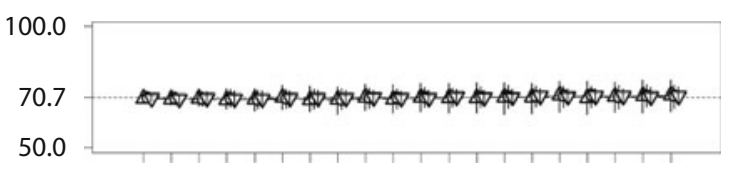

$\begin{array}{llllllllll}0.1 & 0.3 & 0.5 & 0.7 & 0.9 & 1.1 & 1.3 & 1.5 & 1.7 & 1.9\end{array}$

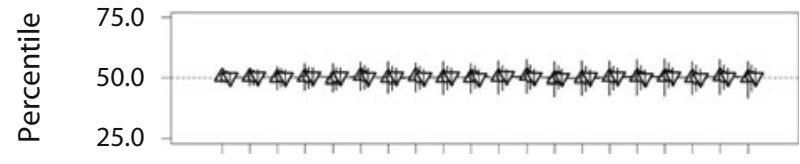

$\begin{array}{llllllllll}0.1 & 0.3 & 0.5 & 0.7 & 0.9 & 1.1 & 1.3 & 1.5 & 1.7 & 1.9\end{array}$

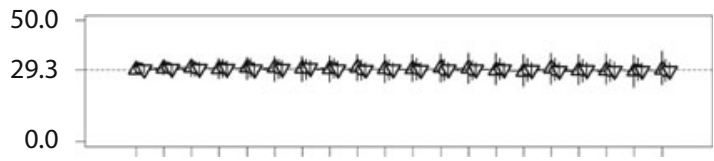

$\begin{array}{llllllllll}0.1 & 0.3 & 0.5 & 0.7 & 0.9 & 1.1 & 1.3 & 1.5 & 1.7 & 1.9\end{array}$

Step Size

Figure 6. Convergences of stochastic approximation (SA) and accelerated stochastic approximation (ASA) with a starting value at the target, based on sample sizes of 25 (triangles), 50 (circles), and 100 (inverted triangles), for the 70.7th (top panels), 50th (middle panels), and 29.3rd (bottom panels) reaction time percentiles, given a fixed $\tau=250 \mathrm{msec}$. Graph conventions are as in Figure 4 . 
TRUD
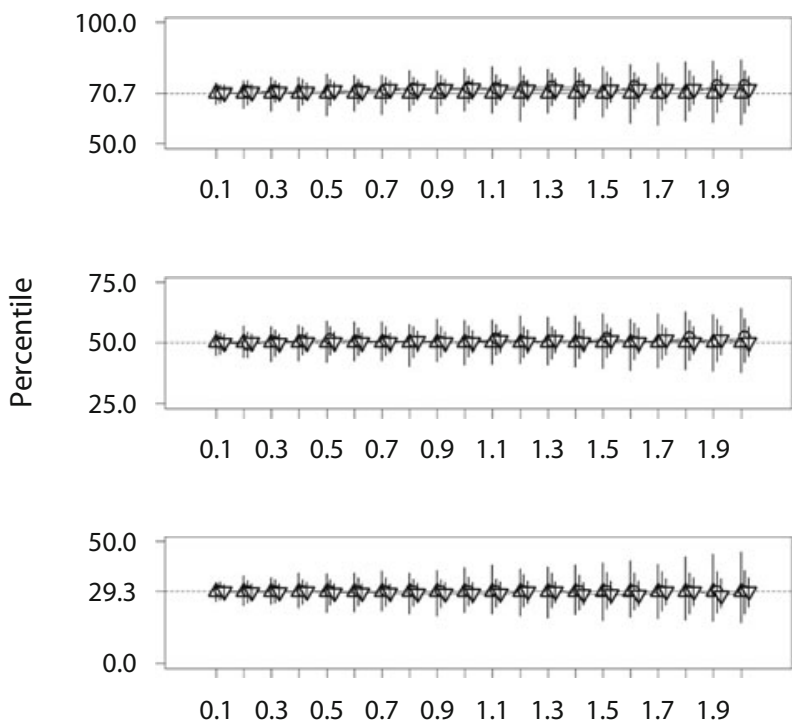

Step Size
BCD
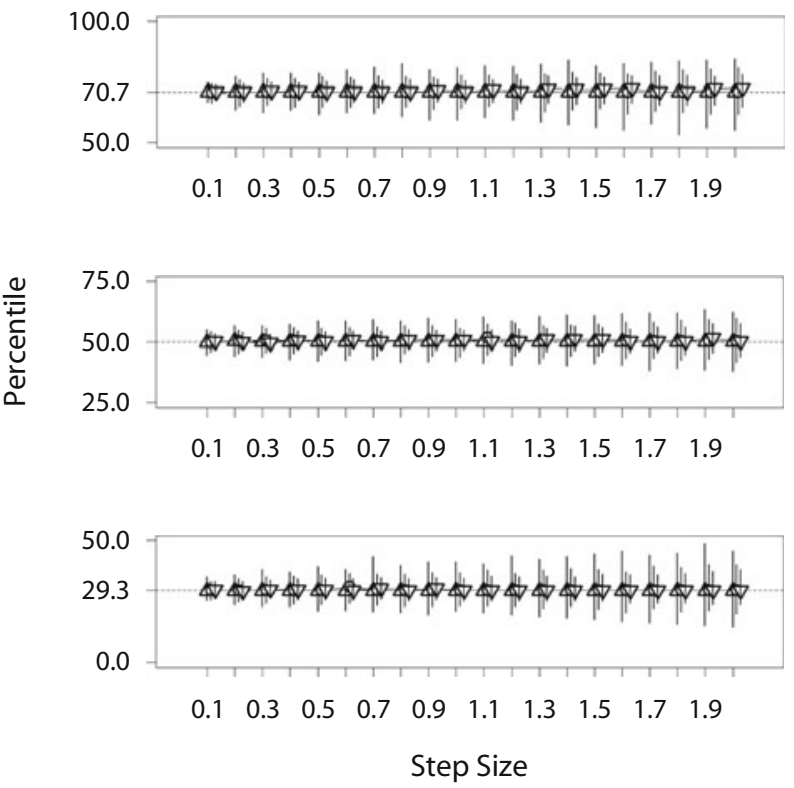

Figure 7. Convergences of transformed up-down (TRUD) and biased-coin design (BCD) with a starting value at the target, based on sample sizes of 25 (triangles), 50 (circles), and 100 (inverted triangles), for the 70.7th (top panels), 50th (middle panels), and 29.3rd (bottom panels) reaction time percentiles, given a fixed $\tau=250 \mathrm{msec}$. Graph conventions are as in Figure 5 .

suggested the (fixed) step size to be in proportion to the standard deviation of the underlying distribution of the psychometric function. For example, Dixon and Mood (1948) recommended an optimal step size to be between $0.5 \sigma$ and $2.4 \sigma$ for a Gaussian-distributed psychometric

SA

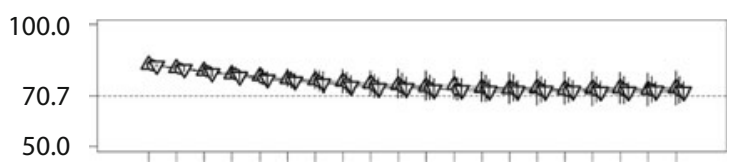

$\begin{array}{lllllllllll}0.1 & 0.3 & 0.5 & 0.7 & 0.9 & 1.1 & 1.3 & 1.5 & 1.7 & 1.9\end{array}$

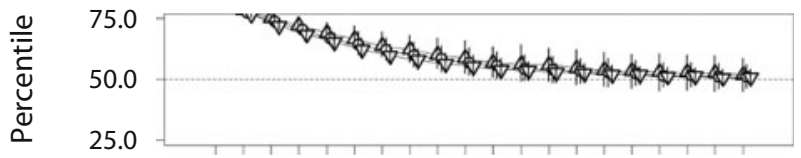

$\begin{array}{llllllllll}0.1 & 0.3 & 0.5 & 0.7 & 0.9 & 1.1 & 1.3 & 1.5 & 1.7 & 1.9\end{array}$

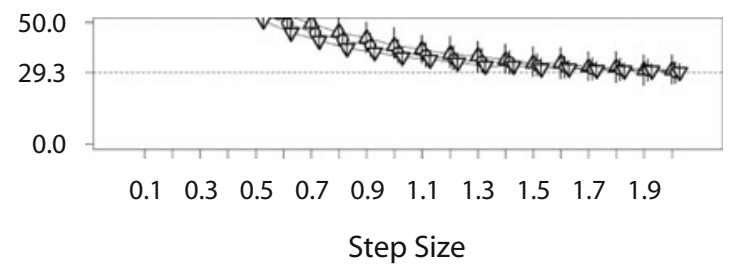

function. In our illustration in Figure 1, an inspection suggests that the standard deviations of those curves are between 0.45 and $0.55 \log$ units, indicating that the ideal step size would be between 0.23 and $1.32 \mathrm{log}$ units, should those curves in Figure 1 be Gaussian distributed. Such a

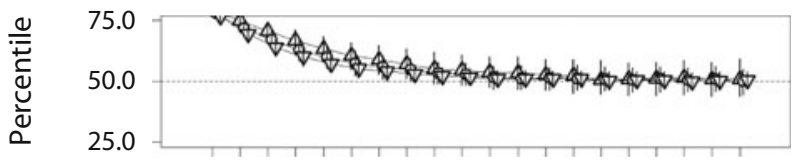

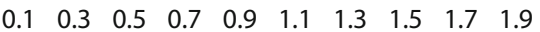

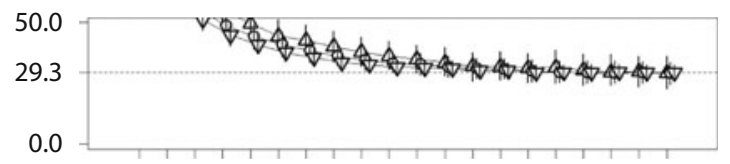

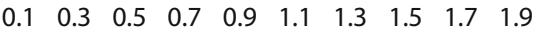

Step Size

Figure 8. Convergences of stochastic approximation (SA) and accelerated stochastic approximation (ASA) with a starting value at $2 \log$ units, based on sample sizes of 25 (triangles), 50 (circles), and 100 (inverted triangles) for the 70.7th (top panels), 50th (middle panels), and 29.3rd (bottom panels) reaction time percentiles/criteria, given a fixed $\tau=250 \mathrm{msec}$. Graph conventions are as in Figure 4 . 
TRUD
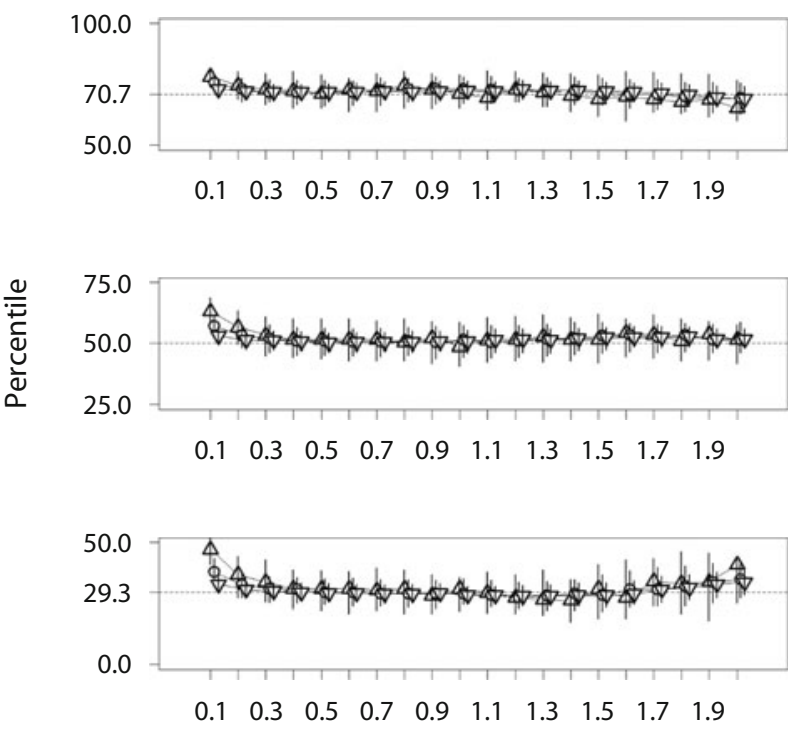

Step Size
BCD
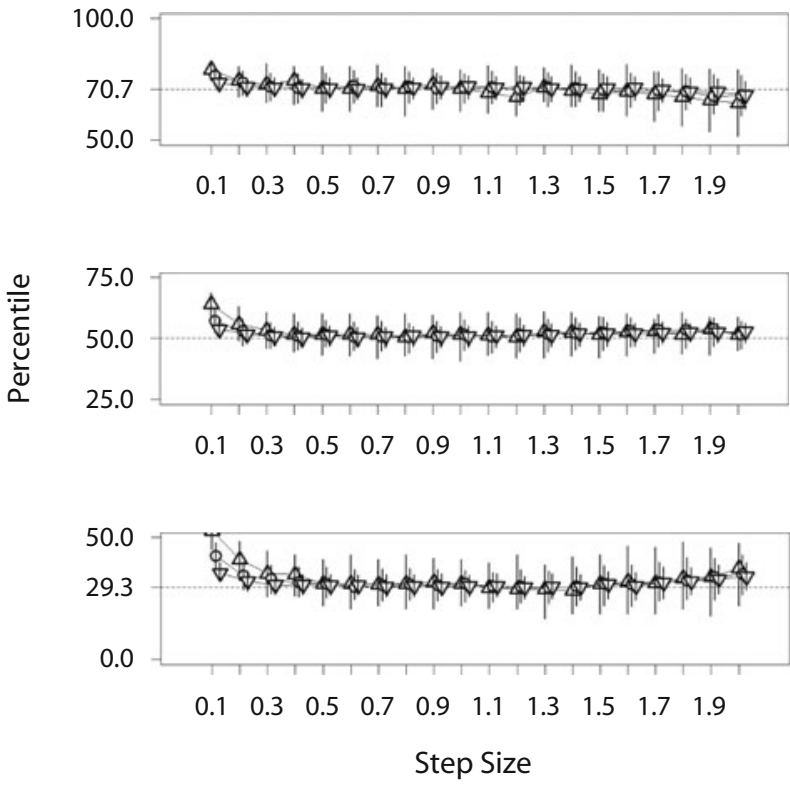

Figure 9. Convergences of transformed up-down (TRUD) and biased-coin design (BCD) with a starting value at 2 log units, based on sample sizes of 25 (triangles), 50 (circles), and 100 (inverted triangles) for the 70.7th (top panels), 50th (middle panels), and 29.3rd (bottom panels) reaction time percentiles/criteria, given a fixed $\tau=\mathbf{2 5 0} \mathbf{m s e c}$. Graph conventions are as in Figure 5.

range of step sizes is fairly covered by our simulation, which ranges from 0.1 to $2 \log$ units. These step sizes are comparable to those used in other studies in psychophysics (see, e.g., Faes et al., 2007; García-Pérez, 1998, 2001), and are adequate to show the pattern of convergences. See also the text in the next section for details.

\section{Results}

Software in R (R Development Core Team, 2008) was used for all of the simulations. The procedure was repeated 1,000 times for each design scenario. In all cases, the deviation of estimations was indexed by the interquartile range (IQR).

We first note that for the three fixed latencies $(225,250$, and $275 \mathrm{msec}$ ) studied in the simulation, in most cases, the sequences converge to the targets. Since the general pattern of convergence is similar for the three latencies, hereafter we will only present the results from fixing $\tau=$ $250 \mathrm{msec}$.

Asymptotic properties. To illustrate, we show in Figure 3 the convergence of sequences for all four methods for the 20.6th, 29.3rd, 50th, 70.7th, and 79.4th RT percentiles/criteria based on $N=150$ trials, with a starting value at $1 \log$ unit. In the figure, every point represents the median of the estimated quantile from 1,000 runs in the simulation. As mentioned above, the starting value of $1 \log$ unit corresponds to the signal that elicits the 51.4th RT percentile, which may or may not be in the neighborhood of the targets, depending on which response percentile/criterion one chooses. It is clear from the figure that with appropriate step sizes, all of the sequences converge to the targets.
Specifically, from Figure 3, one sees that the two families of adaptive methods behave differently for different step sizes. For SA and ASA, the convergence improves steadily as the step size approaches $2 \log$ units. In fact, the convergences (not shown here) are excellent for both SA and ASA for step sizes larger than $2 \log$ units, no matter the starting values. We also notice from the figure that if the starting value is larger than the target, then for small step sizes, there is a positive bias of the estimate; otherwise, the bias is negative.

On the other hand, for TRUD and BCD, the convergence improves with smaller step sizes (except for the $0.1 \log$ unit). The convergence starts to deteriorate (i.e., move away from the target) as the step size approaches $2 \log$ units, and is getting worse (not shown here) for step sizes larger than $2 \log$ units. This is consistent with the results from other fixed-step-size up-down methods for detection and discrimination (see, e.g., García-Pérez, 1998, 2001). One also notes that as the step size increases, the estimate starts to fan out from the target, except for the 50th RT percentile/criterion. That is, for large step sizes, there is a positive bias of the estimate when the criterion is set at a higher region, such as the 70.7 th percentile, and a negative bias when the criterion is set at the lower region such as the 29.3 rd percentile. For other starting values such as -1 and $3 \log$ units, which are extreme for most of the targets, the convergences (not shown here) of both TRUD and BCD for step sizes larger than $2 \log$ units also deteriorate. However, they do not reveal a pattern similar to that in the $1 \log$ unit (starting value) case. Since one of our primary concerns is to study the practical use of the 

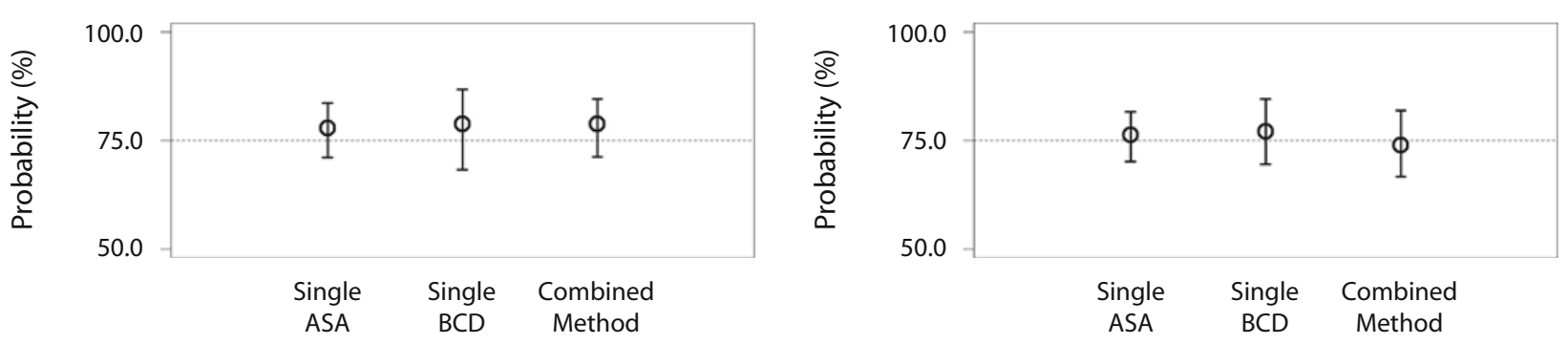

Figure 10. Convergences of accelerated stochastic approximation (ASA), biased-coin design (BCD), and the combined method of ASA and BCD, with starting values at $3 \log$ units (left panel) and $2 \log$ units (right panel), based on a sample size of 25 for the 75 th reaction time percentile/criterion, given a fixed $\tau=250 \mathrm{msec}$. The (initial) step size $c$ for ASA was set at $2 \log$ units, and the (fixed) step size $\delta$ for BCD was set at a $0.5 \log$ unit. For each point, the vertical bar represents the interquartile range.

adaptive methods, we pay special attention to those step sizes between 0.1 and $2 \log$ units.

We will now turn to a more detailed discussion of the comparison of convergences for the four methods.

We will first compare the convergence between SA and ASA. We show in Figure 4 the convergences of estimates (with IQRs) of the two methods using different starting values for the 70.7th, 50th, and 29.3rd RT percentiles/ criteria. The pattern is similar to those for other RT percentiles/criteria. Also, for clarity, we only display the results from the starting values of 3,1 , and $-1 \log$ units. One sees from Figure 4 that in all cases the convergence improves as the step size increases. Furthermore, the convergence is highly correlated with the location of the starting value; when the starting value is close to the target, the sequence converges to the target faster (i.e., with smaller step sizes). One also sees that, except for the starting value of $1 \log$ unit for the median RT criterion, ASA outperforms SA.

Figure 4 also shows that the IQR seems to slightly increase as the step size increases. These results are comparable to the findings from Faes et al. (2007). Overall, our results suggest that ASA with a large initial step size leads to an excellent convergence to the target.

We will now turn to the comparison of the results from the two fixed-step-size up-down methods. One sees from Figure 5 that TRUD and BCD perform similarly well. Note that for the 50th RT percentile/criterion, both TRUD and $\mathrm{BCD}$ are identical to the simple up-down method; thus, the results are identical as well. We mention that in our simulation, for both TRUD and BCD, the average number of reversal points (from 1,000 runs) for each step size is sufficiently large: It ranges from 38 (for the smallest step size, $0.1 \log$ unit for the 70.7th percentile/criterion) to 108 (for the largest step size, $2 \log$ units for the median RT criterion). We also notice that it appears that the number of reversal points is positively correlated with the step size.

From Figure 5, one sees that the convergences of TRUD and BCD are excellent for all small step sizes (except the smallest step size, $0.1 \log$ unit), but that they start to deviate when the step size approaches $2 \log$ units. In particular, at large step sizes, there is a slightly positive bias of estimates for larger RT percentiles/criteria, and a slightly negative bias of estimates for lower RT percentiles/criteria.
One also sees that the IQR seems to increase slightly as the step size increases.

Overall, these results suggest that both TRUD and BCD with a small fixed step size lead to a faster convergence to the target. Having established the asymptotic properties of all four methods, we will now turn to the discussion of the small-sample properties.

Small-sample properties. Small-sample properties are appealing for practical purposes. For example, participants in some clinical populations, such as children with certain visual impairment, may only contribute a rather small amount of data. We systematically varied the total number of trials: $N \mathrm{~s}=25,50$, and 100 . We will discuss two scenarios, one with the starting value at the target, and the other with the starting value away from the target.

The results with starting values at the targets (1.49, 0.97 , and $0.46 \log$ units for the 70.7th, 50th, and 29.3rd RT percentiles, respectively) are displayed in Figure 6 for SA and ASA, and in Figure 7 for TRUD and BCD. It can be seen from both figures that in all cases, the convergences are excellent - even for SA and ASA with small step sizes. The variation of the estimates seems to be larger for TRUD and BCD than for SA and ASA. Furthermore, both figures show that the IQR decreases as the sample size increases.

Overall, we found that all four methods are applicable for small sample sizes, if the starting values are at (or close to) the targets.

It is also informative for one to investigate the smallsample properties when the starting value is away from the target. Here, we use a starting value of $2 \log$ units, which elicits the 84.6th RT percentile for $\tau=250 \mathrm{msec}$. Figure 8 shows the results of SA and ASA. It appears that both methods converge well for large step sizes, with ASA slightly outperforming SA. For the results of TRUD and BCD, one sees from Figure 9 that both methods also converge well in the middle range of the step sizes we have used. However, the IQRs of the estimates for TRUD seem less than those for BCD.

Furthermore, comparing Figure 8 with Figure 9, one sees that the variability of estimates for SA and ASA is smaller than that for TRUD and BCD. This may be due to the fact that for the fixed-step-size up-down methods such as TRUD and BCD, the number of reversal points 
available for estimation is rather few if the sample size is small. In our simulation, with $N=25$, we notice that when the starting point is at the target, for both the $29.3 \mathrm{rd}$ and 70.7th RT percentiles, the (average) number of reversal points in the sequences ranges from 7 (for the step size of $0.1 \log$ unit) to 11 (for the step size of $2 \log$ units); for the 50th RT percentile, it ranges from 12 (for the step size of $0.1 \log$ unit) to 17 (for the step size of $2 \log$ units). When the starting point is at $2 \log$ units, for both the 29.3rd and 70.7th RT percentiles, the number of reversal points ranges from 4 (for the step size of $0.1 \log$ unit) to 11 (for the step size of $2 \log$ units); for the 50th RT percentile, it ranges from 12 (for the step size of $0.1 \log$ unit) to 19 (for the step size of $2 \log$ units). Furthermore, we note that, as in the asymptotic case, the number of reversal points is positively correlated with the step size.

Overall, our small-sample simulation indicates that in all cases, the convergences are good for appropriate step sizes, no matter the initial starting values. This is more evident for the 29.3rd RT percentile/criterion, for which the starting value ( $2 \log$ units, which elicits the 84.6th RT percentile) is far away from those targets; however, all the convergences are fairly good for appropriate step sizes (see the bottom panels in Figures 8 and 9).

We mention that for the stochastic approximation method, the step size is getting smaller and smaller in the sequence. It means in practice that the stimuli called for by the approximation method may not always be available. Also, it might not be practical to find suitable starting values. In such cases, we suggest the following alternative that may bypass these problems. One observes that ASA converges faster for relatively large step sizes, and that $\mathrm{BCD}$ converges faster for relatively small step sizes, suggesting that one can combine the advantages of both methods. For example, for a small sample size, such as 25 trials, one could first run the sequence of ASA using a large step size until the resolution of the stimulus reaches its limit. Suppose this happens on, say, the 5th trial. One then switches the sequence to BCD with that step size for the rest of trials, using the estimate from the 5 th trial as the starting value. ${ }^{9}$ Our simulation has shown that the convergence of this combination of sequences is comparable to the one solely by either ASA or BCD. For example, we illustrate in Figure 10 the comparison of ASA, BCD, and the combined method for the 75th RT percentile based on $N=25$. In this simulation, we used two starting values, 2 and $3 \log$ units, which correspond to 84.6th and 95.4th RT percentiles, respectively. As can be seen, the combined method is feasible, especially when the starting value is away from the target.

\section{Concluding Remarks}

In the present article, we introduced an innovative method for RT experiments that suits our need for obtaining estimates of signal intensities that elicit certain RT percentiles, even with small sample sizes. We extended four nonparametric adaptive methods to the simple RT situation. The four methods are the SA of Robbins and Monro (1951), the ASA of Kesten (1958), the TRUD of Wetherill (1963), and the BCD of Durham and Flournoy $(1994,1995)$.
Similar to the results from psychophysical research in detection and discrimination, we found that the SA and ASA converge faster with larger step sizes, whereas TRUD and BCD converge faster with smaller step sizes. When the starting value is at or close to the target, all four methods show excellent convergences, no matter the sample size. If the starting value is far away from the target, all four methods still show reasonable convergences, even for small sample sizes if the step sizes are set appropriately in their respective ranges. In all scenarios, we found that ASA slightly outperforms SA, and that TRUD and $\mathrm{BCD}$ perform equally well. We also showed that in the case of a small sample size, the combined method of ASA and $\mathrm{BCD}$ is feasible, especially when the starting value is away from the target.

We have shown via simulation that a novel methodology of using nonparametric adaptive methods for the RT situation is stable, even for small sample sizes. Such a methodology can help estimate the signals involved in the near-miss to Weber's law in the framework of the generalized Piéron's law that was mentioned at the beginning of the present article. It also has the potential to be applied to certain clinical groups, such as people with visual impairment, to evaluate and train the speed of discrimination for above-threshold contrasts/objects. Note that since the algorithms needed for the adaptive methods are familiar and easy to implement, this approach may stand in favorable contrast with the hierarchical Bayesian approach that was advocated by Rouder et al. (2005; Rouder et al., 2008).

It remains to be seen whether the proposed methodology is indeed feasible. For example, empirical studies may reveal systematic drifts of data, indicating that the stochastic process is not stationary. An experimental verification is in progress, and this part of our work will be reported elsewhere.

Another ongoing project is to extend the adaptive method for two-choice RT experiments. We mentioned briefly in the introduction that the algorithms used in the present method seem applicable for choice RT in the go/ no-go paradigm..$^{10}$ However, it is not clear how to apply the adaptive method for other two-choice RT experiments in general, because a correct response in the current trial may be followed by an error response in the next trial. This would disrupt the chain in the sequence, which uses a fixed latency as the sole criterion. Of course, one could just ignore those error responses and proceed as if those trials had never occurred. Still, a better solution would be to find an algorithm embedded in the adaptive procedure to incorporate the dependence on error responses into the Markov chain sequence.

\section{AUTHOR NOTE}

The present work was supported by the National Science Council (of Taiwan) Grant NSC-95-2413-H-002-029-MY2 to Y.-F.H. We are grateful to J.-C. Falmagne, who first brought about the idea of applying adaptive methods for RT experiments about 11 years ago in his lab meeting that Y.-F.H. attended at the University of California, Irvine. Y.-F.H. also benefitted from a conversation with Falmagne on the same topic in the summer of 2007. We are much indebted to Chris Doble for his detailed remarks on a previous draft of the manuscript. We also thank Charlie Chubb, Jeff Rouder, and Scott Brown for many helpful comments. Especially, we 
thank Jeff Rouder for providing some examples that enrich the potential application of the present methodology. Address correspondence to Y.-F. Hsu, Department of Psychology, National Taiwan University, No. 1 Sec. 4, Roosevelt Rd., Taipei, Taiwan 106 ROC (e-mail: yfhsu@ntu.edu.tw).

\section{REFERENCES}

Derman, C. (1957). Non-parametric up-and-down experimentation. Annals of Mathematical Statistics, 28, 795-797.

Dixon, W., \& Mood, A. (1948). A method for obtaining and analyzing sensitivity data. Journal of American Statistical Association, 43, 109-126.

Durham, S. D., \& Flournoy, N. (1994). Random walks for quantile estimation. In S. S. Gupta \& J. O. Berger (Eds.), Statistical decision theory and related topics $V$ (pp. 467-476). New York: Springer.

Durham, S. D., \& Flournoy, N. (1995). Up-and-down designs I: Stationary treatment distributions. In N. Flournoy \& W. F. Rosenberger (Eds.), Adaptive designs (pp. 139-157). Hayward, CA: Institute of Mathematical Statistics.

Faes, L., Nollo, G., Ravelli, F., Ricci, L., Vescovi, M., Turatto, M., ET AL. (2007). Small-sample characterization of stochastic approximation staircases in forced-choice adaptive threshold estimation. Perception \& Psychophysics, 69, 254-262.

Falmagne, J.-C. (1985). Elements of psychophysical theory. New York: Oxford University Press.

García-PÉreZ, M. A. (1998). Forced-choice staircases with fixed step sizes: Asymptotic and small-sample properties. Vision Research, 38, 1861-1881.

García-PÉrez, M. A. (2001). Yes-no staircases with fixed step sizes: Psychometric properties and optimal setup. Optometry \& Vision Science, 78, 56-64.

Giovagnoli, A., \& Pintacuda, N. (1998). Properties of frequency distributions induced by general "up-and-down" methods for estimating quantiles. Journal of Statistical Planning \& Inference, 74, 51-63.

Gomez, P., Ratcliff, R., \& Perea, M. (2007). A model of the go/no-go task. Journal of Experimental Psychology: General, 136, 389-413.

Hsu, Y.-F. (2005a). A generalization of Piéron's law to include background intensity and latency distribution. Journal of Mathematical Psychology, 49, 450-463

Hsu, Y.-F. (2005b). On measuring the minimum detection time: A simple reaction time study in the time estimation paradigm. British Journal of Mathematical \& Statistical Psychology, 58, 259-284.

HUANG, C.-B., ZHOU, Y., \& LU, Z.-L. (2008). Broad bandwidth of perceptual learning in the visual system of adults with anisometropic amblyopia. Proceedings of the National Academy of Sciences, 105, 4068-4073.

KaERnBaCH, C. (1991). Simple adaptive testing with the weighted updown method. Perception \& Psychophysics, 49, 227-229.

Kesten, H. (1958). Accelerated stochastic approximation. Annals of Mathematical Statistics, 29, 41-59.

LEEK, M. R. (2001). Adaptive procedures in psychophysical research. Perception \& Psychophysics, 63, 1279-1292.

LEVITT, H. (1971). Transformed up-down methods in psychoacoustics. Journal of the Acoustical Society of America, 49, 467-477.

LUCE, R. D. (1986). Response times: Their role in inferring elementary mental organization. New York: Oxford University Press.

MacLeod, C. M. (1991). Half a century of research on the Stroop effect: An integrative review. Psychological Bulletin, 109, 163-203.

ORON, A. (2007). Up-and-down and the percentile-finding problem. Unpublished doctoral dissertation, University of Washington, Seattle.

Polat, U., Ma-Naim, T., Belkin, M., \& SAGI, D. (2004). Improving vision in adult amblyopia by perceptual learning. Proceedings of the National Academy of Sciences, 101, 6692-6697.

R Development Core Team (2008). $R$ : A language and environment for statistical computing. Vienna: R Foundation for Statistical Computing.

Robisns, H., \& Monro, S. (1951). A stochastic approximation method. Annals of Mathematical Statistics, 22, 400-407.

Rouder, J. N., Lu, J., Speckman, P., Sun, D., \& Jiang, Y. (2005). A hierarchical model for estimating response time distributions. Psychonomic Bulletin \& Review, 12, 195-223.
Rouder, J. N., Sun, D., Speckman, P., Lu, J., \& Zhou, D. (2003). A hierarchical Bayesian statistical framework for response time distributions. Psychometrika, 68, 587-604

Rouder, J. N., Tuerlinckx, F., Speckman, P., Lu, J., \& Gomez, P. (2008). A hierarchical approach for fitting curves to response time measurements. Psychonomic Bulletin \& Review, 15, 1201-1208.

Sahraie, A., Trevethan, C. T., Macleod, M. J., Murray, A. D., Olson, J. A., \& WeISKRANTZ, L. (2006). Increased sensitivity after repeated stimulation of residual spatial channels in blindsight. Proceedings of the National Academy of Sciences, 103, 14971-14976.

Stroop, J. R. (1935). Studies of interference in serial verbal reactions. Journal of Experimental Psychology, 18, 643-661.

Treutwein, B. (1995). Adaptive psychophysical procedures. Vision Research, 35, 2503-2522.

WAGENMAKERS, E.-J., \& Brown, S. (2007). On the linear relation between the mean and the standard deviation of a response time distribution. Psychological Review, 114, 830-841.

Wetherill, G. (1963). Sequential estimation of quantal response curves. Journal of the Royal Statistical Society: Series B, 25, 1-48.

Wetherill, G., Chen, H., \& Vasudeva, R. (1966). Sequential estimation of quantal response curves: A new method of estimation. Biometrika, 53, 439-454.

Zhou, Y., HuanG, C.-B., Xu, P., TAo, L., Qiu, Z., Li, X., ET AL. (2006). Perceptual learning improves contrast sensitivity and visual acuity in adults with anisometropic amblyopia. Vision Research, 46, 739-750.

\section{NOTES}

1. Further discussion is delayed until the Concluding Remarks section.

2. Hsu (2005b) motivated the derivation with a race-type model of competing (hypothetical) "agents" (each having a Weibull-distributed finishing time), in which the exponent $\beta$ can be derived. See also Rouder et al. (2008) for a related but different theoretically motivated development, in which they explored a similar power law on the covariate as one of the probable formulations.

3. It is granted that such a formulation may not fit all the situations. For example, a process-driven model may derive that $T_{\pi}(i)=R+$ $\left(-i^{k} \log \pi\right)^{-1 / \beta}$, which is outside the generalized Piéron's law.

4. In psychophysical study of detection and discrimination, the simple up-down method is customarily called a " 1 -up/1-down" (rule) because one incorrect (and correct) response on the current trial results in an "up" (and a "down,") of the stimulus by $\delta$ on the next trial.

5. We also have investigated other fixed-step-size up-down methods for estimating nonmedian reaction time (RT) quantiles, such as the weighted up-down method (Kaernbach, 1991). However, our simulation study has shown that the weighted up-down method is not an optimal algorithm in the RT framework. We do not pursue this method further in the present article.

6. The biased-coin design (BCD) is similar in spirit to the one proposed by Derman (1957), but has some desired properties (Giovagnoli \& Pintacuda, 1998; Oron, 2007). We have studied both designs via simulations and have found that the results from BCD are superior to those by Derman. Thus, we do not pursue Derman's method further in the present article.

7. Note that those curves are not psychometric functions in the usual sense.

8. Our present study (not shown here) revealed that other distributions displaying a similar right-skewed shape, such as the Gamma, yielded asymptotic and small-sample properties in the simulation similar to those for the Weibull. Hereafter, we will only report the results using the Weibull.

9. Falmagne (1985, pp. 228-229) also mentioned a similar recommendation of combining accelerated stochastic approximation (ASA) and the (fixed-step-size) up-down method of Derman (1957) for the psychophysical study of detection and discrimination.

10. For a recent review on the comparison of go/no-go and twochoice RT experiments in the context of lexical decision, see Gomez et al. (2007)

(Manuscript received December 28, 2008; revision accepted for publication April 21, 2009.) 